\title{
Principal Component Analysis and Factor Analysis
}

\author{
Therese Leinonen
}

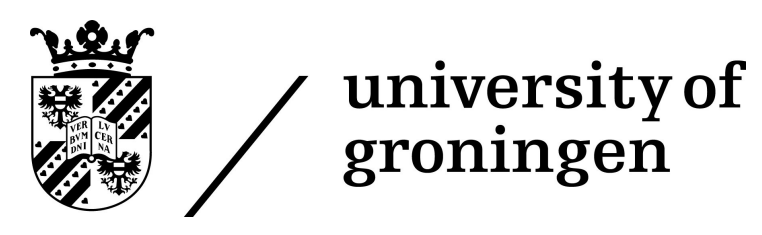

Seminar in Statistics and Methodology, 25th February, 2009 


\section{Overview}

- Introduction

- Basic mathematics behind PCA and FA

- Background: on acoustic measures for vowel quality

- Example: PCA on Bark filtered vowel spectra

- PCA vs. FA 
- factor analysis (FA) and principal component analysis (PCA) are data reduction methods

- by analyzing the correlation between variables in a data set the variables can be reduced to a smaller amount of factors (FA) or principal components (PCA)

- both methods give a set of loadings an a set of scores

- loadings are correlations between original variables and extracted factors/components

- scores are values each data item gets on the extracted factors/components after data reduction 


\section{Basic mathematics behind PCA and FA}

- starting point: a correlation matrix or a variance-covariance matrix

- by determining the eigenvalues and eigenvectors of the matrix variables that correlate highly can be clustered together on components(/factors)

- the eigenvectors (=loadings) are ordered by eigenvalue, highest to lowest, which gives the components in order of significance

- by choosing the most significant components the eigenvectors of these can be used to derive the scores (=reduced data set) for each subject

- the interpretability of the solutions can be enhanced by different rotation techniques 


\section{Background: on acoustic measures for vowel quality}

- measuring formant frequencies is the traditional way of analyzing vowel quality acoustically

- formants = peaks in vowel spectra resulting from resonance in the vocal tract

- the first two formants (F1 and F2), corresponding well with vowel height and backness, are usually enough to distinguish vowels from each other

- problem with formant measurements: automatic detection of peaks in the spectrum not reliable 


\section{spectrum}

spectrogram vowel
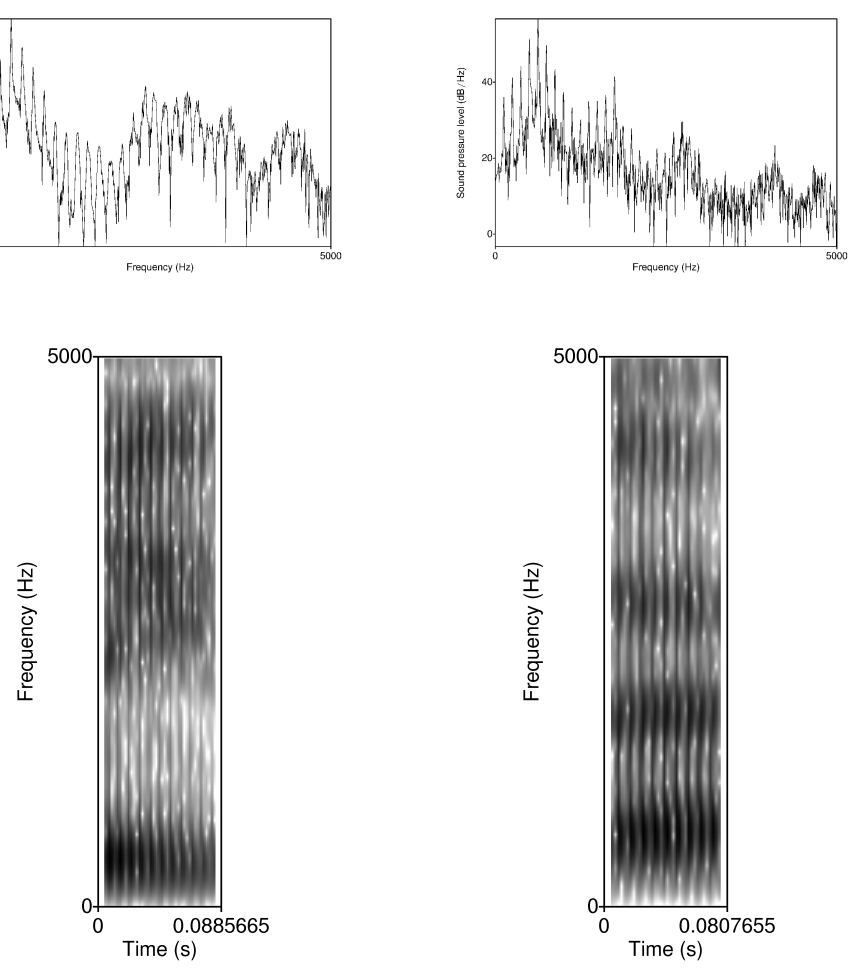

[i]
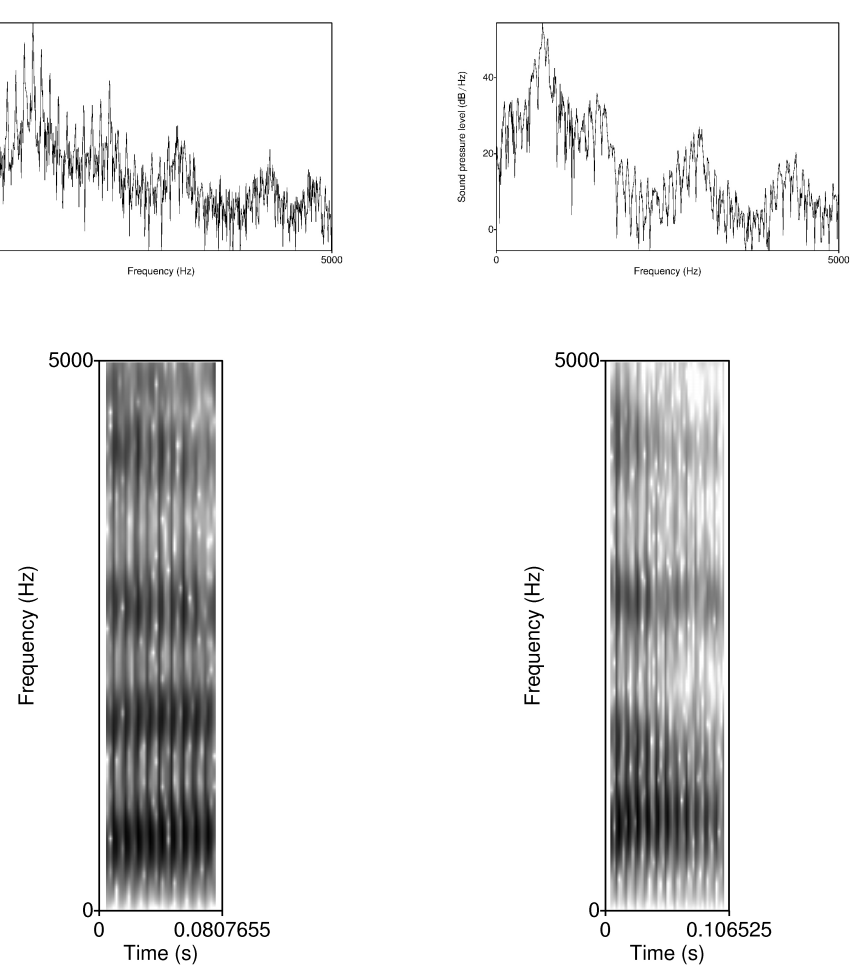

[æ]

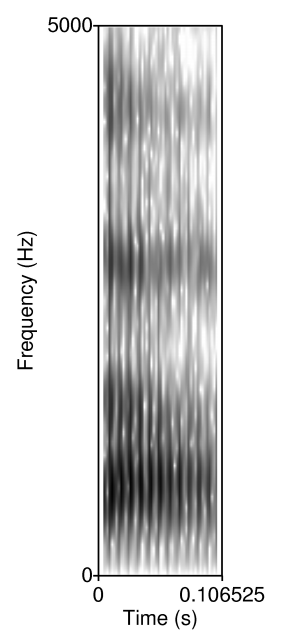

[a]
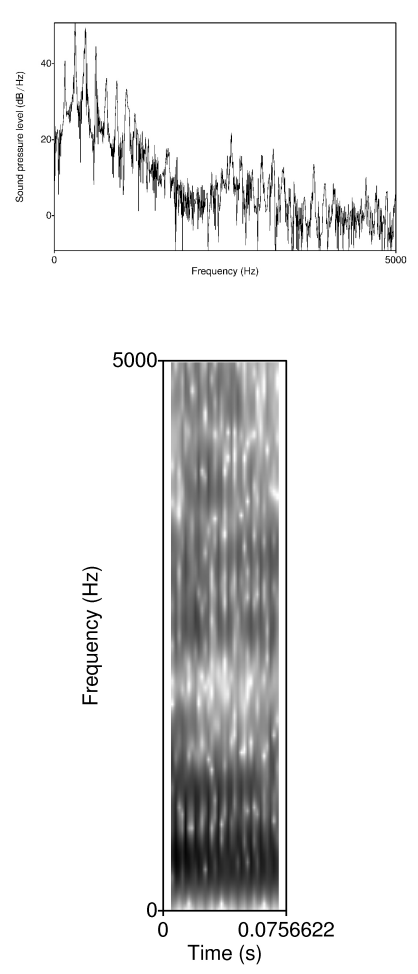

[u] 


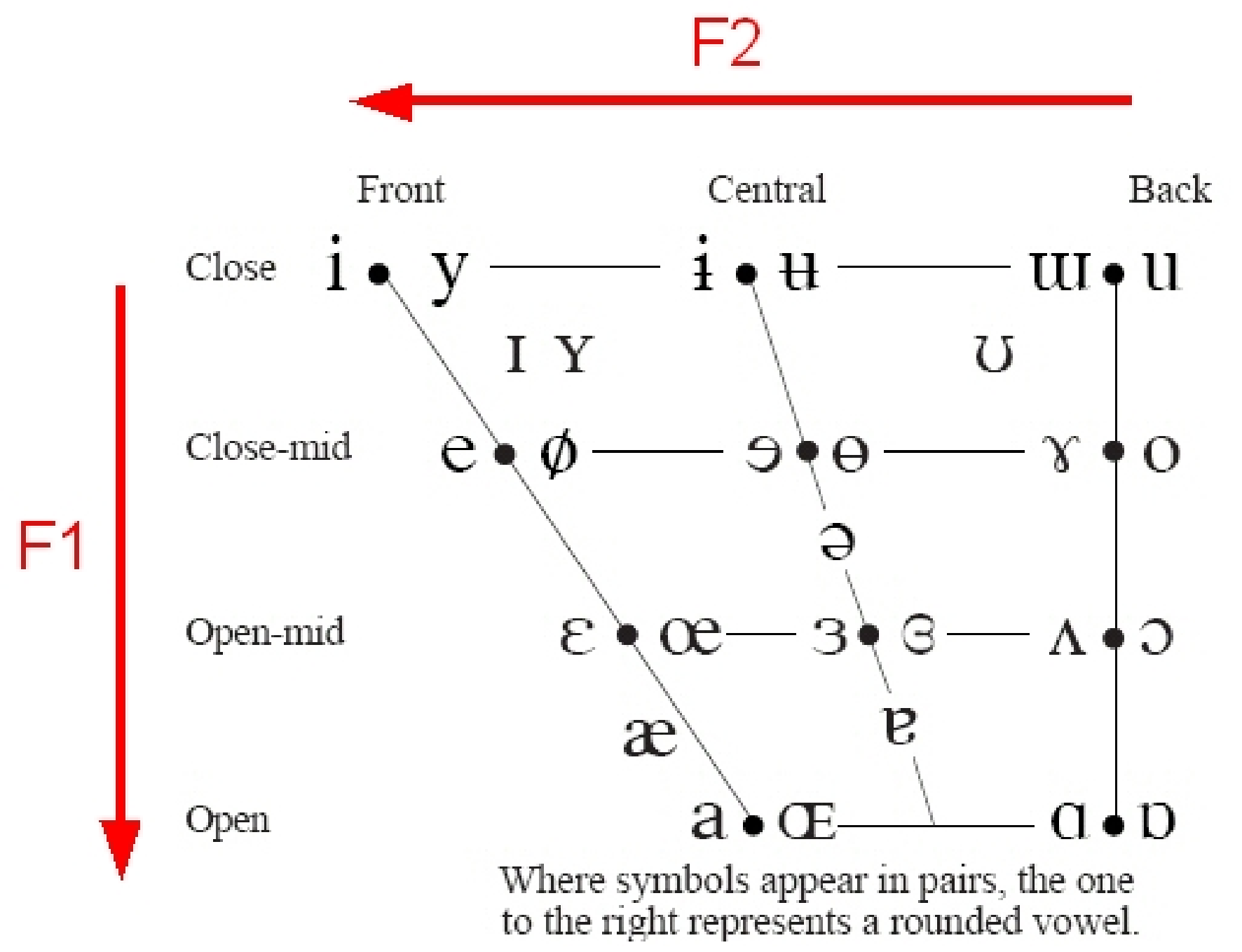




\section{Example: PCA on Bark filtered vowel spectra}

- method introduced by Pols, Tromp and Plomp (1973), recently applied for analyzing sub-phonemic variation in Dutch vowels by Jacobi (2008)

- alternative to formant measurements, can be fully automated

- vowel spectra filtered up to 17 Bark with every filter covering 1 Bark, mean intensity $(\mathrm{dB})$ per filter band measured for each vowel pronunciation => every pronunciation is described by 17 variables

- since we know that vowel quality can generally be described with much fewer variables (2?) we use PCA for data reduction 


\section{How many components should be extracted?}

- scree plot

- eigenvalues higher than 1

- research design

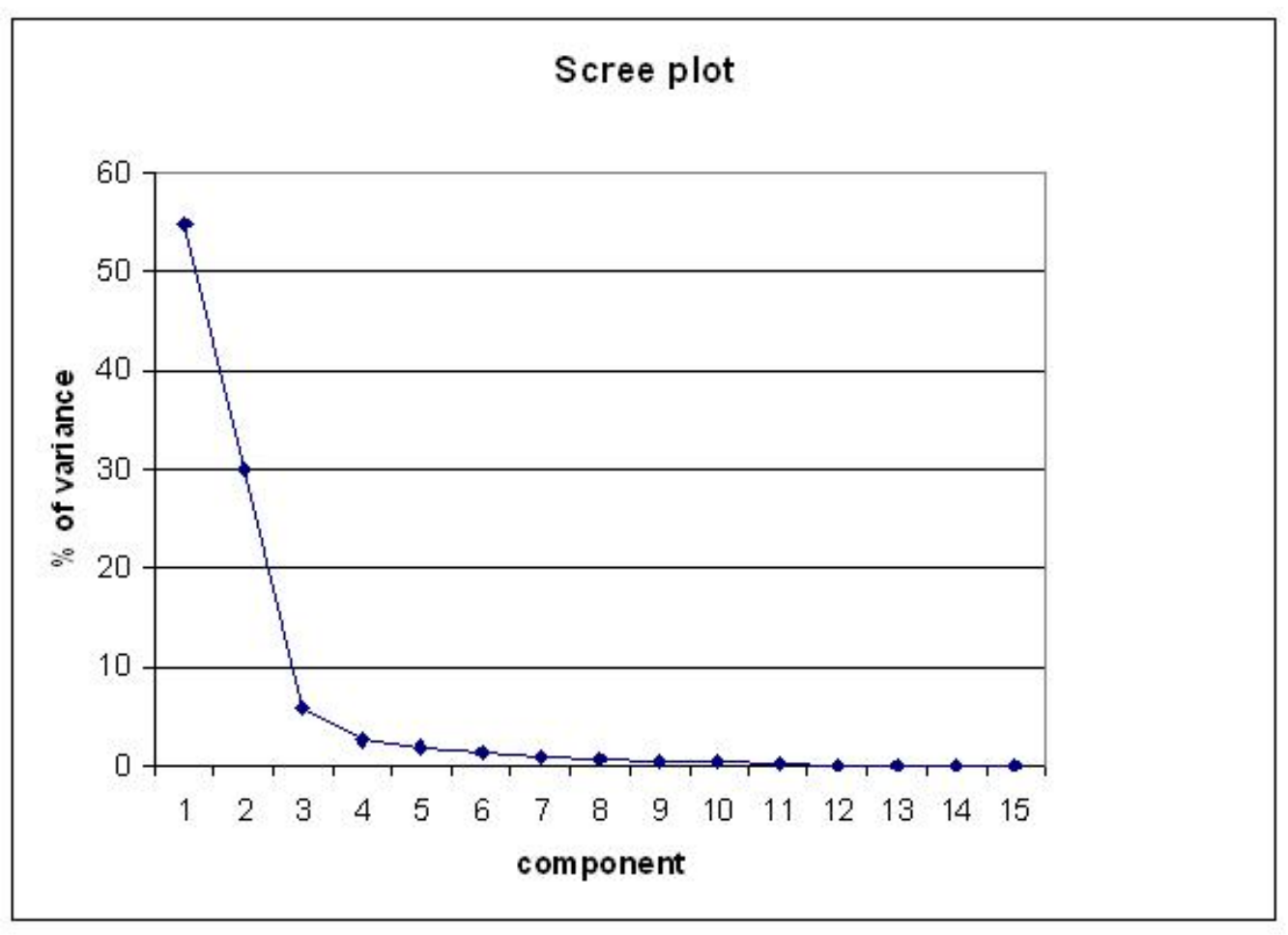




\section{Loadings}

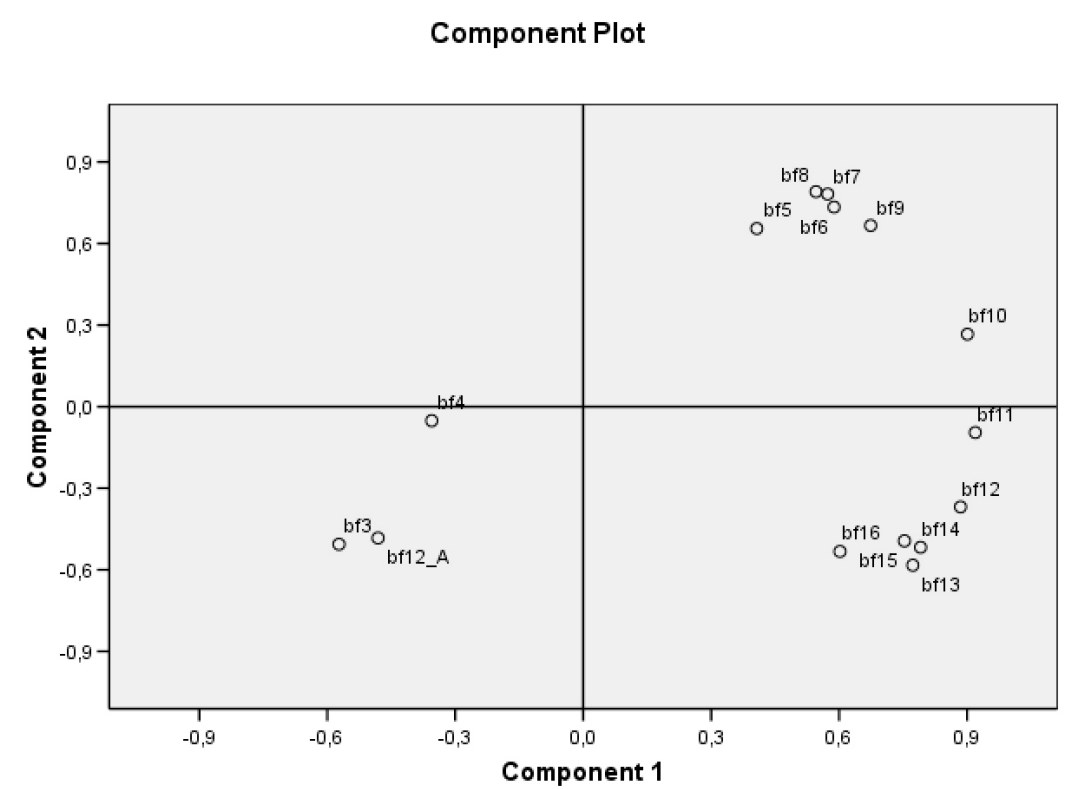

Component Plot in Rotated Space

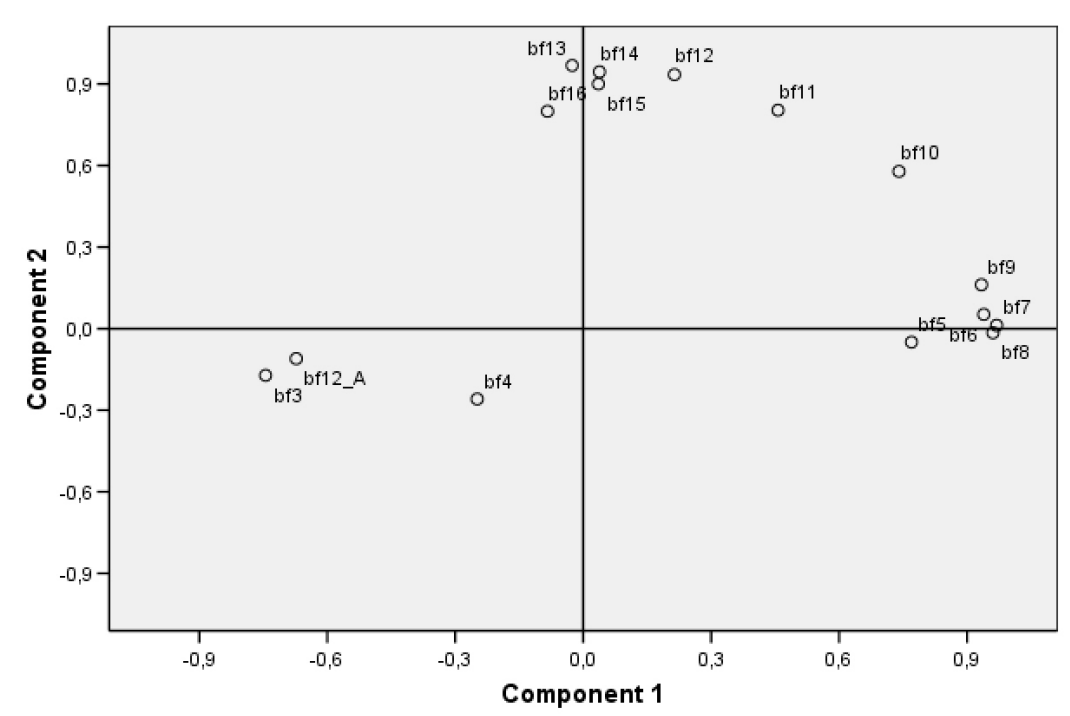




\section{Scores}

Scores PCA

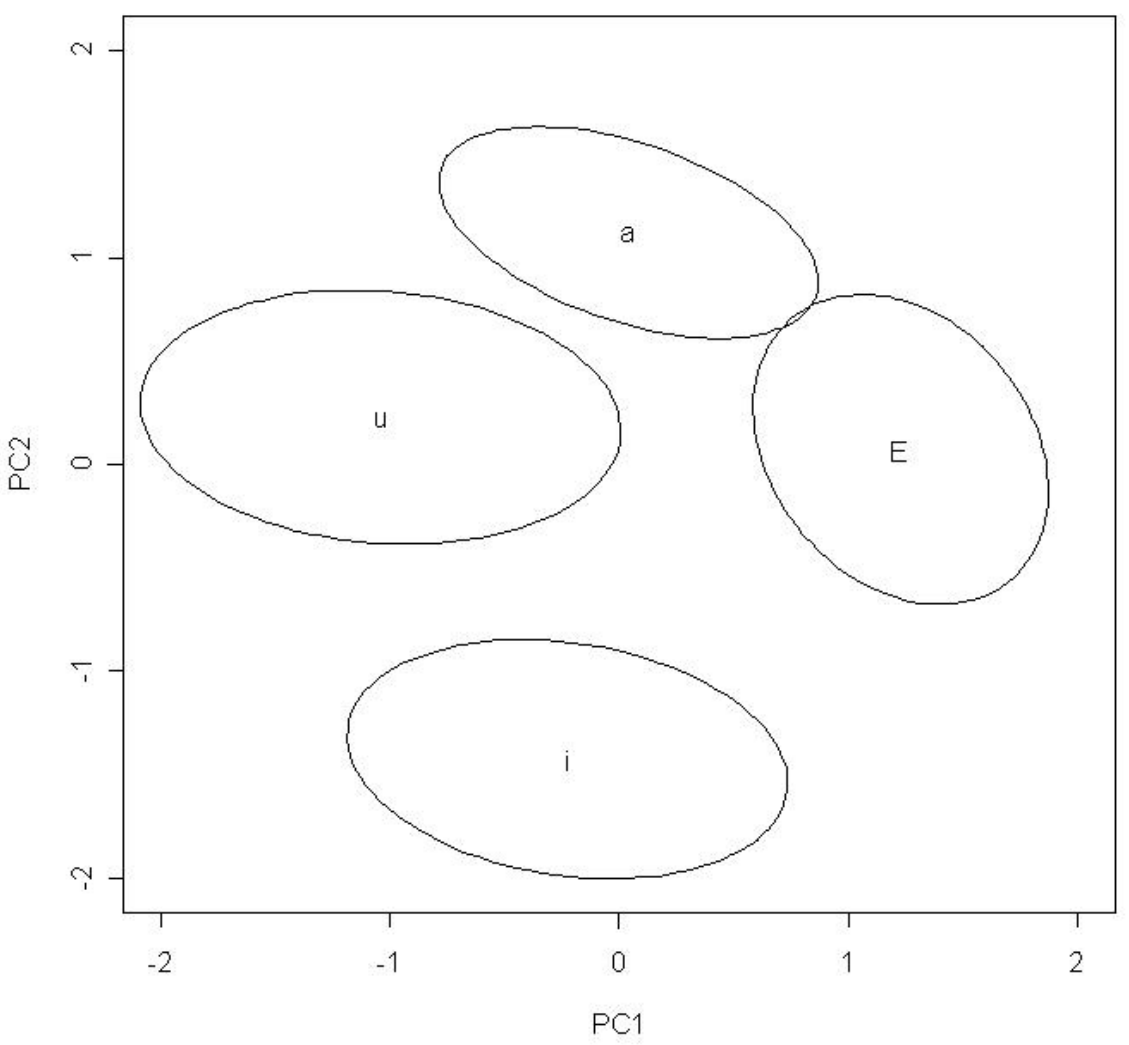

Scores PCA with varimax rotation

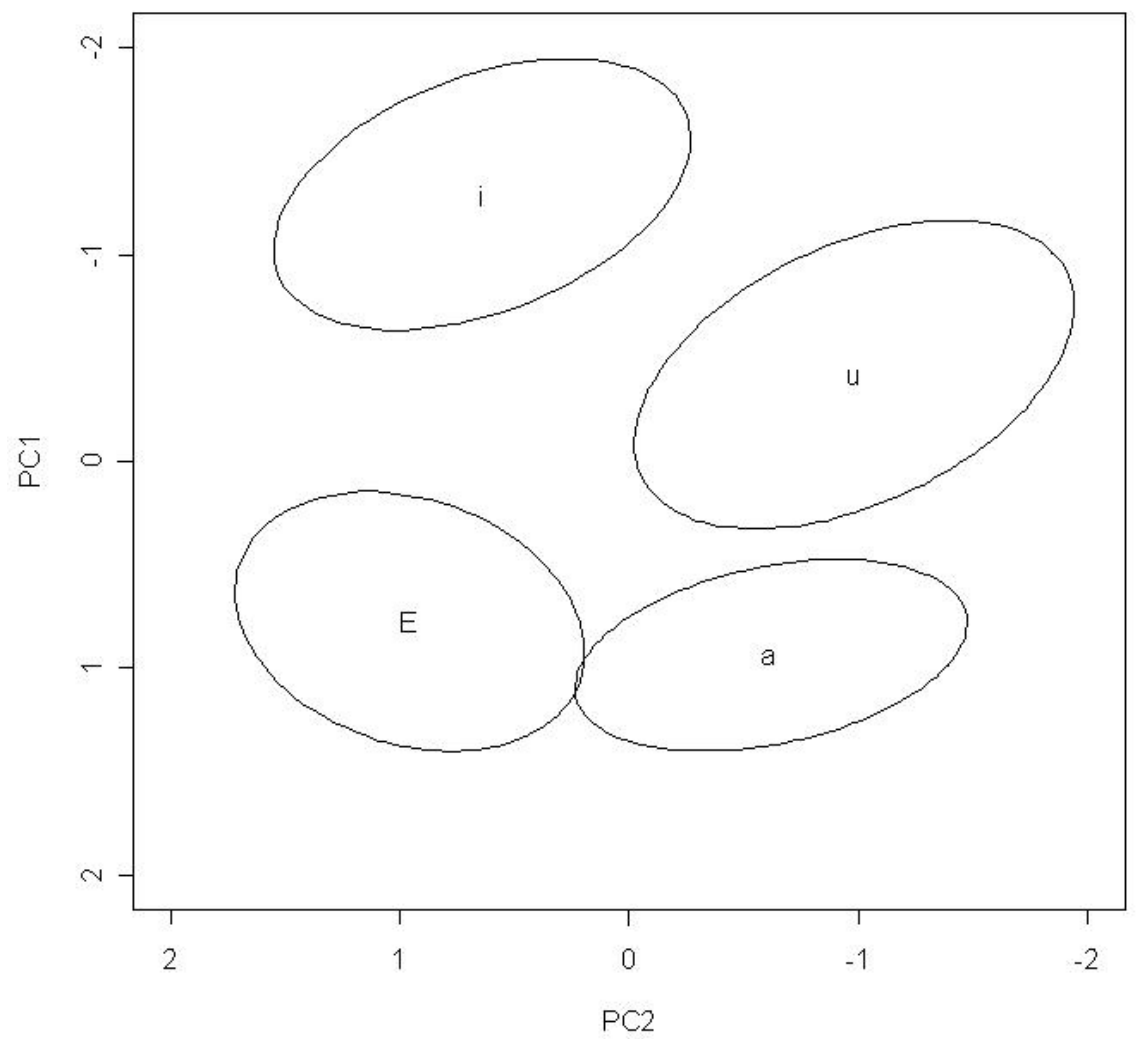


- PCA analyzes all variance present in the data set, while FA analysis only common variances (=uncontaminated by unique and error variability) $=>F A$ less sensitive to noise in the data

- in PCA the first component explains as much as possible of the total variance, the second component as much as possible of the still remaining variance etc. => when interpreting components one should bare in mind that most of the variance has been explained by previous components

- PCA should be used if you want an empirical summary of the data, FA if the study is based on assumed underlying factors (Tabachnik and Fidell 2007)

- however, if the study includes a large number of variables $(>30)$ and communalities are high $(>0.7)$ different solutions with PCA and FA are unlikely (Field 2005) 


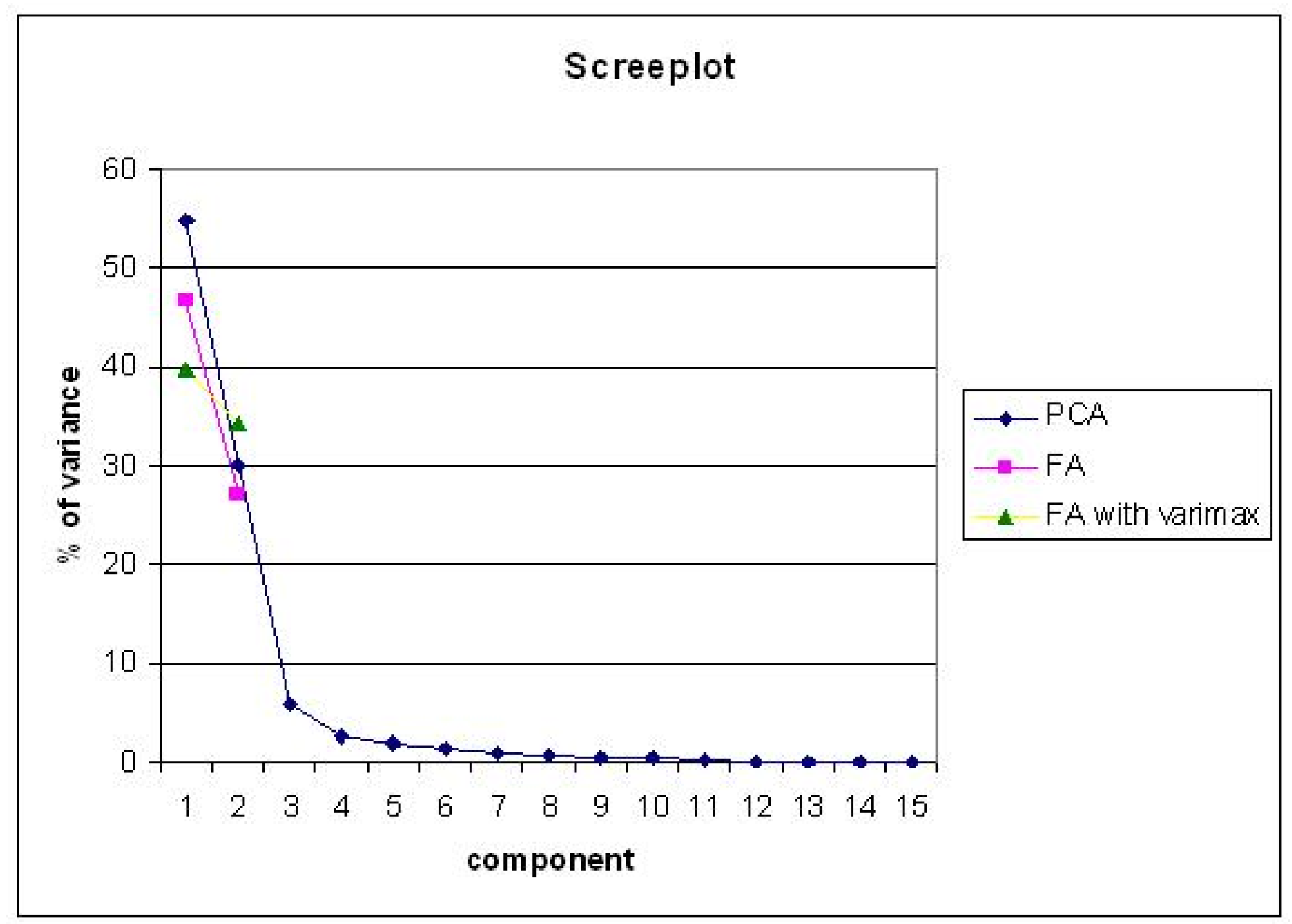




\begin{tabular}{c|c|c} 
& $\mathrm{F} 1$ & $\mathrm{~F} 2$ \\
\hline $\mathrm{F} 1$ & 1 & -0.115 \\
\hline $\mathrm{F} 2$ & -0.115 & 1
\end{tabular}

\begin{tabular}{c|c|c} 
& PC1 & PC2 \\
\hline F1 & 0.606 & 0.589 \\
\hline F2 & 0.420 & -0.727
\end{tabular}

\begin{tabular}{c|c|c} 
& PC1 rotated & PC2 rotated \\
\hline F1 & 0.866 & 0.122 \\
\hline F2 & -0.385 & 0.741
\end{tabular}

\begin{tabular}{c|c|c} 
& factor1 rotated & factor2 rotated \\
\hline F1 & 0.848 & 0.123 \\
\hline F2 & -0.394 & 0.752
\end{tabular}


1. interpret the loadings and scores of the analysis as such

2. use the results in subsequent analyses (MANOVA, multiple regression etc.) 


\section{References}

Field, A.(2005), Discovering Statistics Using SPSS, 2nd edn, SAGE, London.

Jacobi, I.(2008), On Variation and Change in Diphthongs and Long Vowels of Spoken Dutch, $\mathrm{PhD}$ thesis, Universiteit van Amsterdam.

Pols, L. C. W., Tromp, H. R. C. and Plomp, R.(1973), Frequency analysis of Dutch vowels from 50 male speakers, Journal of the Acoustical Society of America 53, 1093-1101. Tabachnik, B. G. and Fidell, L. S.(2007), Using Mulitvariate Statistics, 5th edn, Pearson. 